\title{
Enhancing the Odd Peaks Detection in OFDM Systems Using Wavelet Transforms
}

\author{
Ahlam Damati', Omar Daoud ${ }^{2}$, Qadri Hamarsheh ${ }^{3}$ \\ ${ }^{1}$ Department of Electrical Engineering, Philadelphia University, Amman, Jordan \\ ${ }^{2}$ Department of Communications and Electronics Engineering, Philadelphia University, Amman, Jordan \\ ${ }^{3}$ Department of Computer Engineering, Philadelphia University, Amman, Jordan \\ Email: odaoud@philadelphia.edu.jo
}

Received 5 June 2016; accepted 18 July 2016; published 21 July 2016

Copyright (C) 2016 by authors and Scientific Research Publishing Inc.

This work is licensed under the Creative Commons Attribution International License (CC BY).

http://creativecommons.org/licenses/by/4.0/

c) (i) Open Access

\begin{abstract}
This work aims to study the effect of unwanted peaks and enhance the performance of wireless systems on the basis of tackling such peaks. A new proposition has been made based on wavelet transform method and its entropy. Signals with large peak-to-average power ratio (PAPR) will be examined such as the ones that are considered as the major Orthogonal Frequency Division Multiplexing (OFDM) systems drawbacks. Furthermore, aspatial diversity Multiple-Input MultipleOut-put (MIMO) technology is used to overcome the complexity addition that could arise in our proposition. To draw the best performance of this work, a MATLAB simulation has been used; it is divided into three main stages, namely, MIMO-OFDM symbols' reconstruction based on wavelet transform, a predetermined thresholding formula, and finally, moving filter. This algorithm is called Peaks' detection based Entropy Wavelet Transform; PD-EWT. Based on the simulation, and under some constrains such as the bandwidth occupancy and the complexity structure of the transceivers, a peak detection ratio has been achieved and reaches around 0.85 . Comparing with our previously published works, the PD-EWT enhances detection ratio for 0.25 more peaks.
\end{abstract}

\section{Keywords}

Wavelet Transform, Entropy, MIMO, OFDM, PAPR

\section{Introduction}

The overwhelming huge data due to the highly demand for the various wireless and cellular system's applications attract the researchers' interest to handle these effects on the wireless systems. Thus, and during the last two decades, their attentions have been focused on the combination between the Orthogonal Frequency Division 
Multiplex (OFDM) modulation technique and the Multiple-Input Multiple-Output (MIMO) technology.

Therefore, we are talking a data rate of around more than 100 Mbps for such systems. The OFDM systems use the parallel transmission, while the MIMO technologies have been employed to reduce the effect of the rich scattering environments.

Moreover, the OFDM has been adopted at the both wireless and wired application to the high data rates as significant advantages over the conventional ones, and shows robustness to multipath fading and a greater simplification of channel equalization.

Furthermore, the multiple antennas have been employed to support the extraordinary data rates due to the rapid growth of the wireless systems and to make use of the rich scattered environments [1]-[5]. The MIMO technologies that could be used for this purpose are either the spatial multiplexing or BLAST [6].

OFDM technique is considered as a multi-carrier system that utilizes a parallel processing technique and allowing the simultaneous transmission of data on many closely spaced, orthogonal sub-carriers. This is attained by making use of the Inverse fast Fourier transforms (IFFT) and fast Fourier transform. However, the peak-toaverage power ratio (PAPR) is found as a major deficiency of the OFDM signal, which limits the efficiency of the non-linear devices such as the power amplifiers, mixers, and analog to digital converters. Therefore, the wavelet transform method has been used to tackle the effect of such deficiency as will be discussed in section two [7]. Previously in [8], another proposition of PAPR reduction technique has been addressed based also on wavelet transformation technique. It was based on De-noise the OFDM using some DWT, after that defining an adaptive threshold to limit those peaks, and finally replace these peaks and valleys using an average filter. This algorithm gives an enhancement around 0.65 of reducing the peaks production. The PAPR could be defined as shown in Equation (1) on the maximum power of the OFDM symbol and its average power as:

$$
\text { PAPR }=10 \log _{10}\left[\frac{P_{\text {peak }}}{P_{\text {avg }}}\right]
$$

$P_{\text {peak }}$ is the maximum power of an OFDM symbol, and $P_{\text {avg }}$ is the average power. The PAPR can be reformulated as given in (2). $T$ is the symbol duration, $x(t)$ is the OFDM symbol at time, $t . X_{n}$ is the data modulating the $n^{\text {th }}$ sub-carrier and $f_{o}$ is the nominal subcarrier frequency spacing. Moreover, the average power of the OFDM symbol presented in Equation (2) will be given in Equation (3):

$$
\begin{gathered}
\text { PAPR }=\frac{\left|\frac{1}{\sqrt{N}} \sum_{n=0}^{N-1} X_{n} \mathrm{e}^{j 2 \pi f_{0} n t}\right|^{2}}{\frac{1}{N T} \int_{0}^{N T}\left|\frac{1}{\sqrt{N}} \sum_{n=0}^{N-1} X_{n} \mathrm{e}^{j 2 \pi f_{0} n t}\right|^{2} \mathrm{~d} t} \\
P_{\text {avg }}=\frac{1}{T} \int_{0}^{T}\left(\sum_{v=0}^{N-1} c_{v}^{2}\right) \mathrm{d} t
\end{gathered}
$$

Here, $c_{v}$ is the magnitude of the modulated data. For the sake of simplicity; $\left|c_{v}\right|=1$, which can be attained by using BPSK modulation with channel coding less techniques at the interval of $\tau \in[0, T]$. This will result a direct relationship between the average power and the total number the IFFT points, $N$. It is clearly shown from Equation (4) as follows:

$$
P_{\text {avg }}=N \frac{1}{T} \int_{0}^{T} c_{v}^{2} \mathrm{~d} t=N,
$$

From [4] and based on the mathematical modelling that was used to combat the effect of the PAPR; the PAPR will be decreased if the average power of the OFDM symbol is decreased. The following flowchart shows the previously proposed technique in Figure 1.

Another technique to compare with could be found in Figure 2. It can be summarized by the following steps:

1) Read a segment of the OFDM signal.

2) Denoise the OFDM signal from additive white Gaussian noise (AWGN) using wavelets technique [8]. In this step the unwanted random addition to a wanted signal is removed using the following sub steps:

- Applying discrete wavelet transform DWT to the noisy signal. 


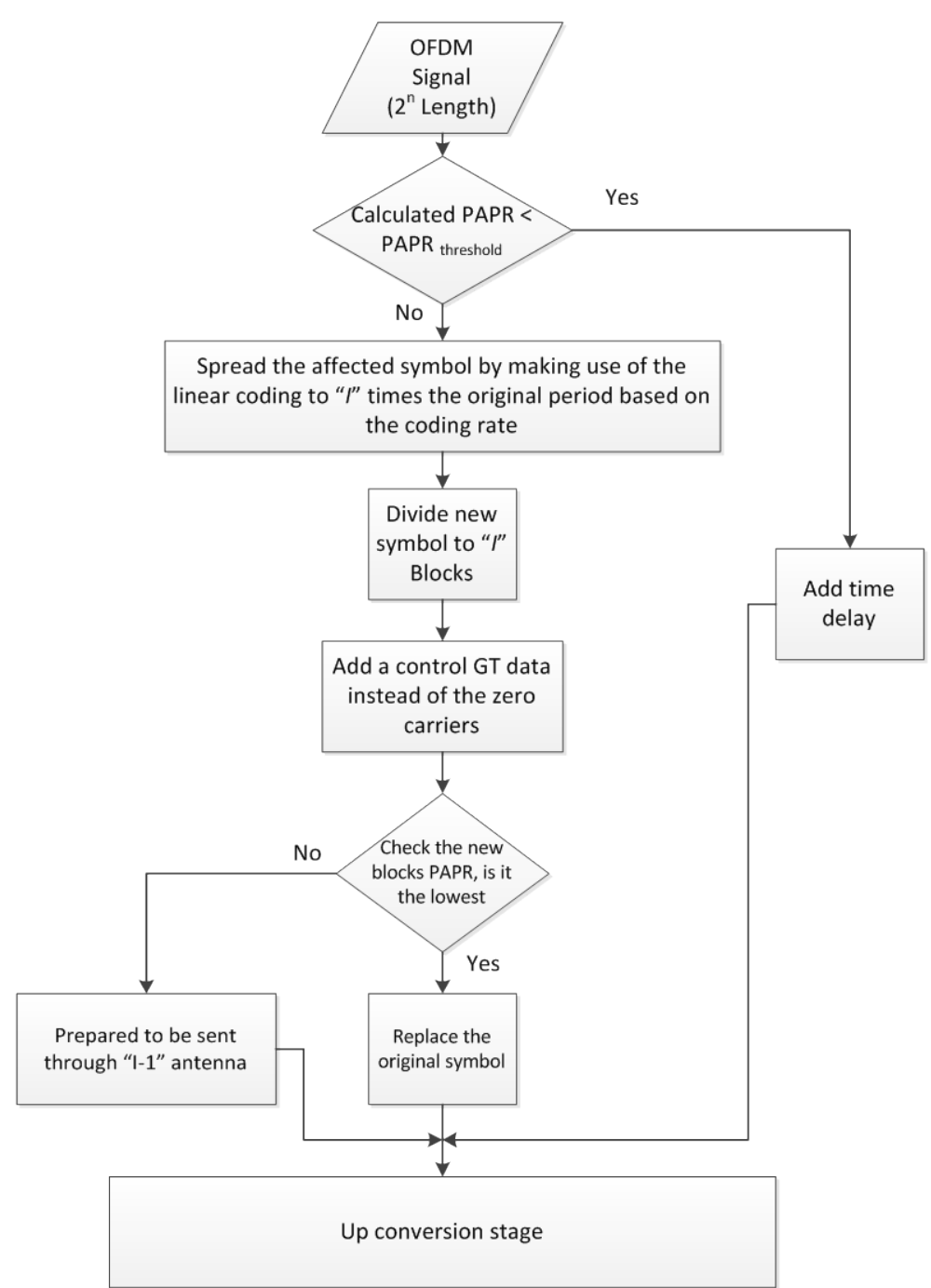

Figure 1. The flowchart of the algorithm based linear coding [9].

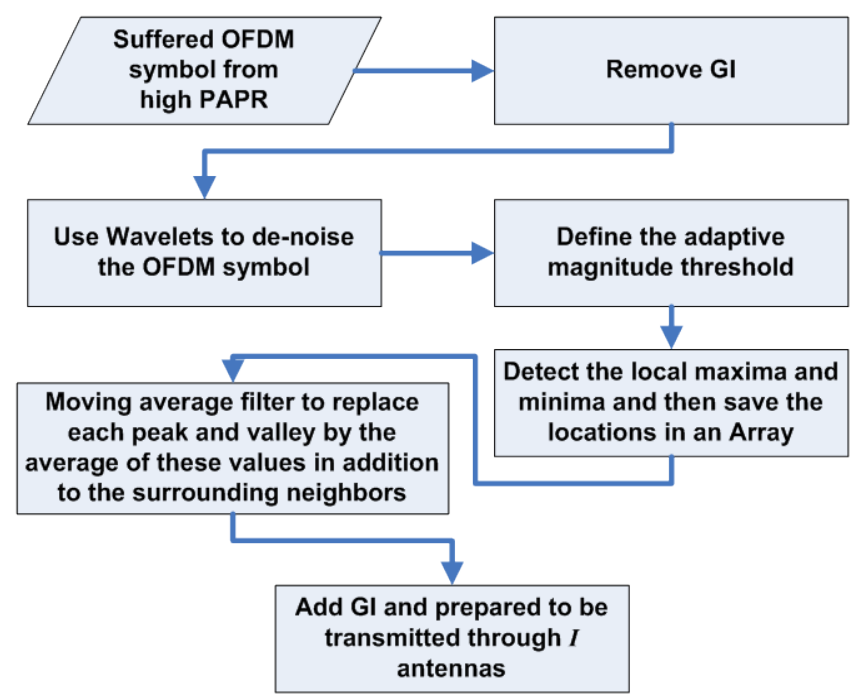

Figure 2. The flowchart of the algorithm based on the wavelet [8]. 
- Applying soft thresholding operator (wavelet shrinkage) [8] to highlight large values of wavelet coefficients which almost correspond to the OFDM signal and suppress small values which correspond to noise.

- Applying inverse discrete wavelet transform IDWT to the thresholded wavelet coefficients to reconstruct a denoised OFDM signal.

In this work, the wireless systems' performance will be drawn for the PD-EWT and compared to the previously published work in [8] [9]. This performance will be based on the BER. It is known that either the wrong detection or the noisy channels will cause burst error and then special protection is necessary.

Let us define first the received OFDM symbol as shown below in Equation (5)

$$
\hat{S}=s_{0}+s_{1}
$$

where $s_{0}$ is the useful information, $s_{1}$ is the interference signals. After that, the SINR expression could be deduced as

$$
\operatorname{SINR}=\frac{E\left\{\left|s_{0}\right|^{2}\right\}}{E\left\{\left|s_{1}\right|^{2}\right\}}
$$

Then, the BER comes from defining the relationship between the bit error probabilities with the SINR. Thus, a mapping function could be defined through the link level simulation with the needed channel. Making use of the definition that is found in [10] which is based on Chernoff Union bound.

The rest of paper is organized as follows; the introduced structure of the proposed algorithm in the MIMOOFDM wireless system is defined in Section 2, the simulation results are presented in Section 3, while the last section summarizes the conclusion.

\section{The Description of the Used PD-EWT in the Wireless Systems}

Figure 3 shows the description of the used wireless system; MIMO-OFDM system, and it is divided into three main stages; OFDM stage, PD-EWT stage and the MIMO stage.

From Figure 1, the transmission layer contains three different stages; the OFDM stage, the proposed O-EWT; which proposed to overcome the effect of the PAPR, and the MIMO stage. For the OFDM stage, it consists of three main blocks as shown in Figure 4.

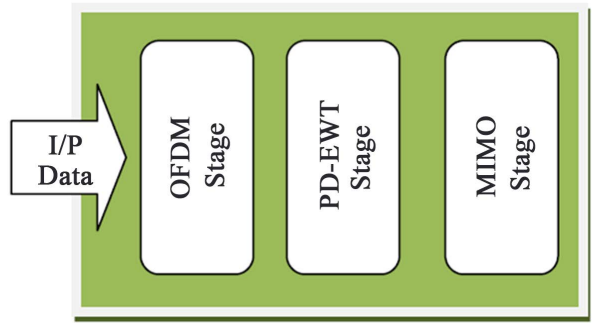

Figure 3. The proposed work block diagram.

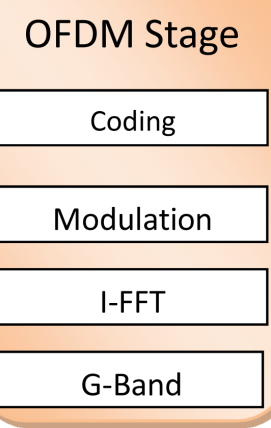

Figure 4. The OFDM stage structure. 
In Figure 4, the turbo encoder of 1/2 coding ratio is used in the coding block, 16 QAM for the modulation block, the overall throughput expressed in terms of bits/symbol for OFDM symbols, and generated by applying the IFFT. After the IFFT stage and due to the coherent addition of the independently modulated subcarriers to produce OFDM symbol, a large PAPR ratio could appear.

The generated OFDM signal will pass through the second stage which is capable of detecting the high PAPR peaks and overcoming their effect. The whole work in this stage could be divided into four blocks as shown in Figure 5. After that, the achieved results will be compared with our previously published work [8] [9].

The continuous wavelet transform (CWT) is attained as a sum of time signals multiplied by a scaled and a shifted version of small wavy functions that are proficiently limited duration with an average of zero. Moreover, and if these scaled versions have been generated based on powers of two, therefore the discrete wavelet transform (DWT) will be obtained. In addition to the wavelet transforms that are based on the decomposition high and low pass filters namely wavelet packet transform is the WP. A pair of low and high pass filters is used to recognize two sequences capturing dissimilar frequency sub-band features of the original signal. These sequences are then decimated (dissembled by a factor of two). It was indicated by many works that WP features have better presentation than the DWT [11].

In [12], the authors define the mathematical meaning of the Entropy for a discrete random variable $X$ as:

$$
H(X)=-\sum P\left(X=a_{i}\right) \log \left(\sum P\left(X=a_{i}\right)\right)
$$

$H$ is the entropy, $a_{i}$ are the discrete random variable, $X$, possible values. This equation reflects the disorder degree that the variable acquires. Then, the discrete wavelet decomposition for sampled values of the signal $S(t)$ could be written as:

$$
S(t)=\sum_{j=-N}^{-1} \sum_{k} C_{j}(k) \psi_{j, k}(t)
$$

The signal $S(t)$ is given by the sampled values, $C_{j}(k)$ is the wavelet coefficient and limited to following frequency interval $2^{j-1} \omega_{s} \leq|\omega| \leq 2^{j} \omega_{s}$.

Moreover, the wavelet entropy could be defined in terms of wavelet coefficients relative wavelet energy as follows:

$$
P_{j}=\left(\frac{E_{j}}{E_{\text {total }}}\right)
$$

$E_{j}$ is the energy at each $j$ resolution level, $E_{\text {total }}$ is the sum of $E_{j s}, \quad j=N, \cdots, 1$. After defining the meaning of the wavelet transforms, we can conclude that the scope of this work is the use of DWT while it could be further emphasized in the future to cover the WP. Thus, the proposed algorithm starts with scanning the resultant $x(t)$ that is defined previously. This signal will be processed as follows:

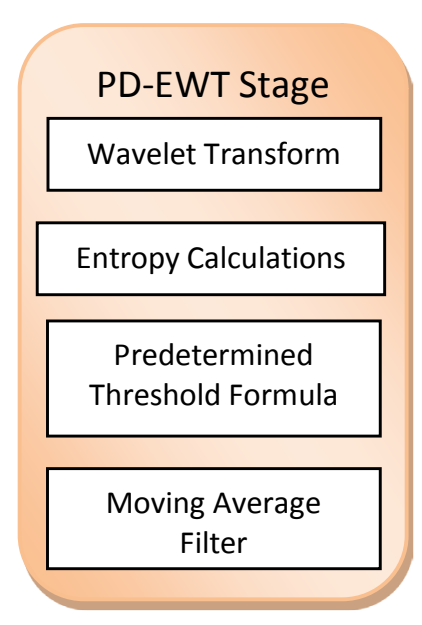


- The preprocess stage:

- Remove the noise from a signal using wavelet technology.

- Perform P-level Haar wavelet decomposition of a signal $(\mathrm{P}=8)$.

- Construct the approximations, CAP and the details CDP.

- Zero Crossing mechanism

- The entropy Calculation

- Case studies based on decomposition process using the depicted flowchart in Figure 6, the results of these case studies clearly depicted in Figure 7.

- Case Study 1: Detect true and false local extremes points using all details coefficients (CDP1-CDP8)

- Case Study 2: Detect true and false local extremes points using details coefficients (CDP1,CDP2)

- Case Study 3: Detect true and false local extremes points using all details coefficients except (CD3, CD7 and CD8).

- Thresholding process

- Moving average (MA) filter.

In this section, a new technique has been proposed to allocate peaks in the OFDM signal based on the entropy wavelet packets. It is clearly seen in Table 1, the entropy of the original signal is about 100.4268. After dividing the used signal into eight levels using the wavelet packets, the entropy has been divided into the range of 0.68262 to 74.2432 . Therefore and based on the entropy we can use the best combination that results the best peaks allocation process.

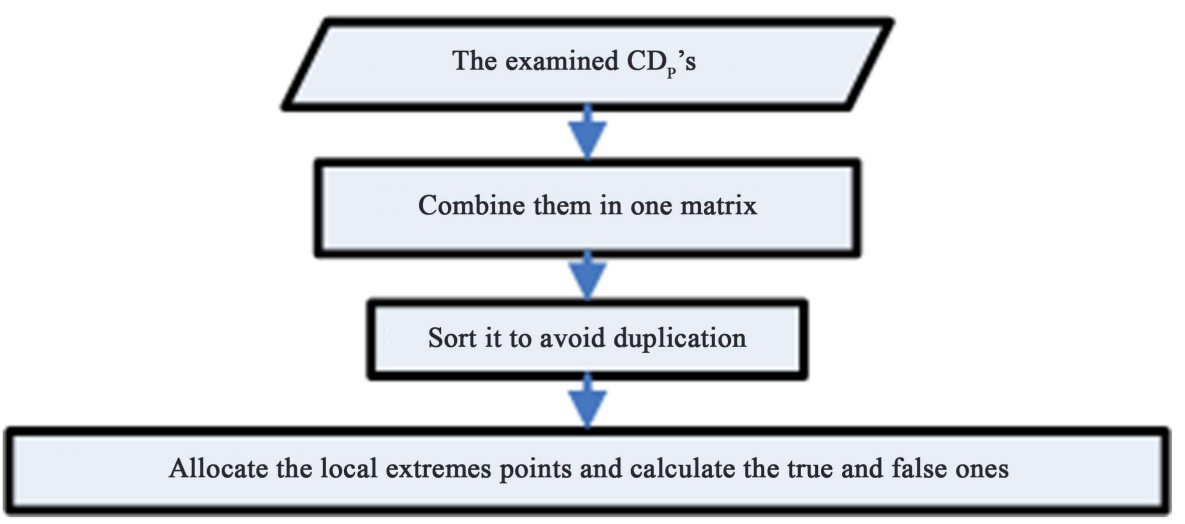

Figure 6. The used procedure flowchart.

Table 1. Packet's entropy values.

\begin{tabular}{|c|c|c|c|c|c|}
\hline \multirow{2}{*}{ P level } & \multicolumn{4}{|c|}{ Entropy } & \multirow{2}{*}{$\begin{array}{l}\text { Decomposition } \\
\text { Acceptance }^{*}\end{array}$} \\
\hline & $\mathrm{CDp}$ & CAp & Summation & Original Signal & \\
\hline 1 & 13.3924 & 60.8508 & 74.2432 & \multirow{8}{*}{100.4268} & Accepted \\
\hline 2 & 26.5319 & 32.917 & 59.4489 & & Accepted \\
\hline 3 & 16.3553 & 20.7162 & 37.0715 & & Not Accepted \\
\hline 4 & 8.2519 & 9.7128 & 17.9647 & & Accepted \\
\hline 5 & 6.3765 & 3.2822 & 9.6587 & & Accepted \\
\hline 6 & 1.6068 & 0.93369 & 2.5405 & & Accepted \\
\hline 7 & 1.541 & 0.54864 & 2.0897 & & Not Accepted \\
\hline 8 & 0.62314 & 0.059486 & 0.68262 & & Not Accepted \\
\hline
\end{tabular}

( $^{*}$ Accepted, if the sum of the entropies at certain level is less than the entropy above this level. 


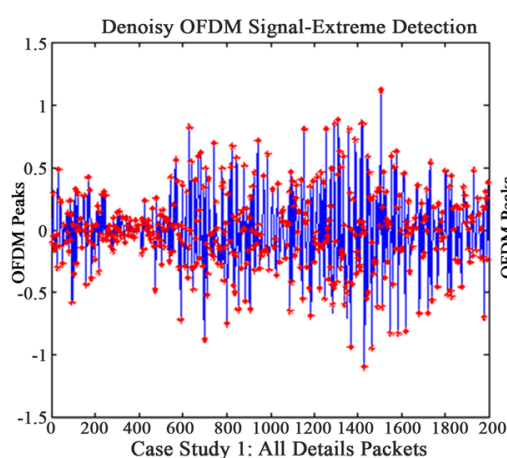

(a)

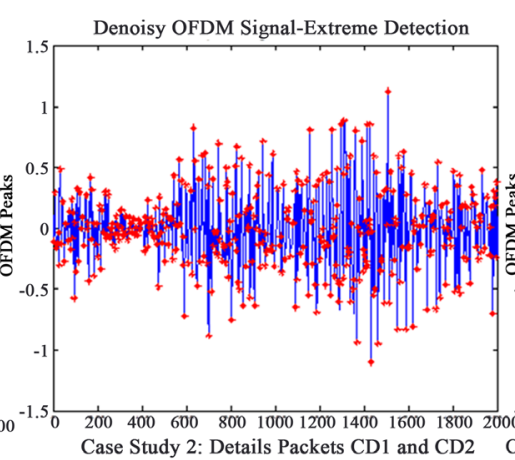

(b)

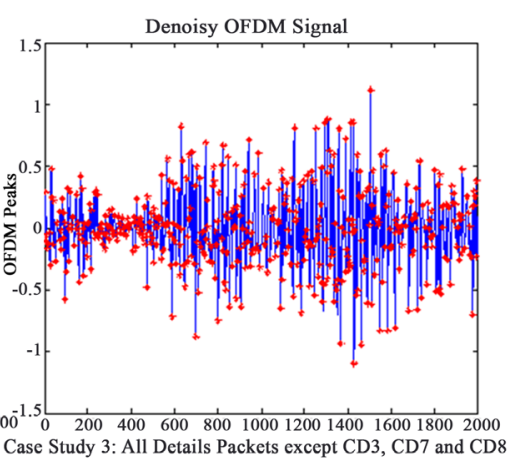

(c)

Figure 7. Odd peaks detection based on the different three case studies.

\section{Simulation Results and Discussion}

The MATLAB simulation program was performed and limited to the use of

- Theoretical randomly generated test data,

- Simple linear convolutional encoder,

- 16-Quadrature Amplitude Modulation (16 QAM) and Binary-Phase Shift Keying (BPSK),

- $\quad$ IFFT size of 256.

The novelty in this work rises from the way of dealing with the entropy of the wavelet coefficients to determine the peaks in the OFDM signal before the transmission. For checking the system performance, two main key factors will be studied; the bit error rate (BER) and the complementary cumulative distribution function (CCDF) curves for the processed OFDM signal.

As a comparison, Table 2 demonstrates powerfulness of the proposed work over either the work that are found in the literature or our previously published works; It is in the range of $15 \%-81 \%$ as an extra PAPR reduction ratio.

Table 2 shows clearly the improvement of the proposed work comparing either to our previously published work or to the SLM technique that found in the literature. The achieved reduction rate varies between $8 \%$ $81.62 \%$ based on the selected case study and the used technique.

Figure 8 and Figure 9 show the simulation part that is based on the CCDF and BER curves with different modulation techniques. These results check the performance of our system from reducing the PAPR problem point of view for two different modulation techniques; 16QAM and BPSK, respectively. These figures compare the threshold value against the probability that the PAPR will exceed the threshold value. From these figures the reduction improvements are clearly shown over what have been achieved in the literature for the conventional MIMO-OFDM systems.

The CCDF plots that are shown in Figure 8 show that the probability of the peaks that will exceed the $16 \mathrm{~dB}$ could be reduced to be $3.9 \times 10^{-3}$ while it was $53 \times 10^{-2}$. Moreover, it shows an extra $15 \%$ reduction percentage over the PAPR combating technique that is in [9]. Figure 9 shows the BER curves for different modulation technique; BPSK to confirm the reliability of the proposed work in combating the PAPR problem. Thus, the performance of the MIMO-OFDM based FFT is still better than either that of the conventional PAPR reduction techniques or our previously published work in [8] [9]. At $20 \mathrm{~dB}$ threshold, the BER curve for the conventional MIMO-OFDM system shows a reduction from $48 \times 10^{-2}$ to $36 \times 10^{-2}$.

\section{Conclusions}

A new proposition has been made in this paper; PD-EWT. This work introduces a new OFDM transceivers design. It is based on allocating the peaks and valleys of OFDM signal to be analyzed. In the PD-EWT, the entropy of DWT has been analyzed and used to specify those peaks. The allocated peaks then will be processed using a special thresholding algorithm to overcome its effect.

Making use the analytical derivation of this technique to build a MATLAB simulation, which ease the study of its feasibility. This is in order to enhance the MIMO-OFDM wireless systems' performance even in a condensed Multipath channel using two different modulation techniques; BPSK and 16 QAM. 


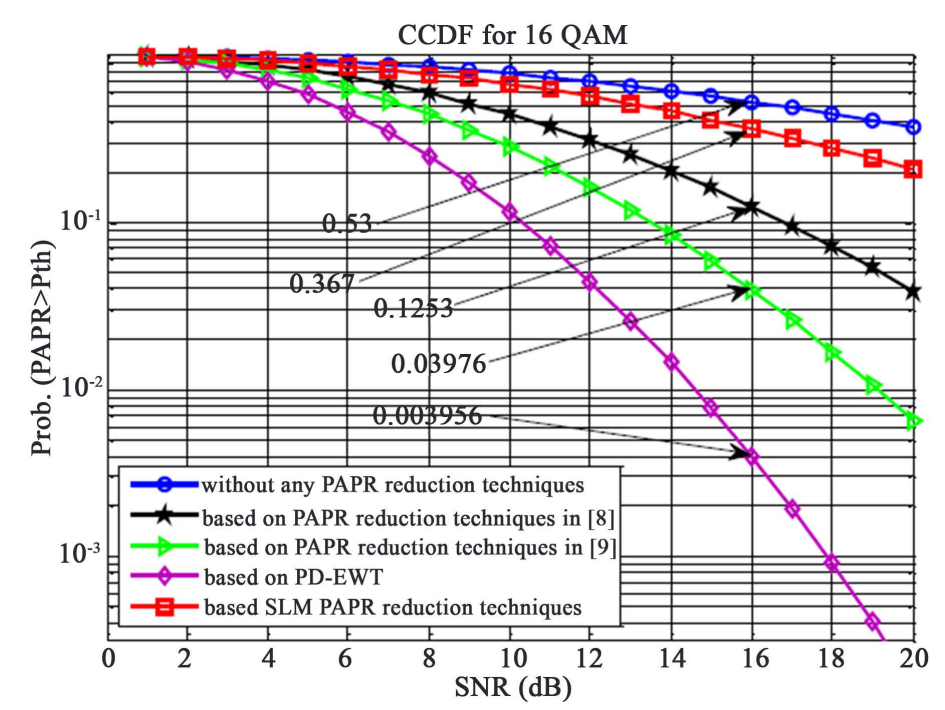

Figure 8. Compared CCDF curves for different reduction technique using case study II.

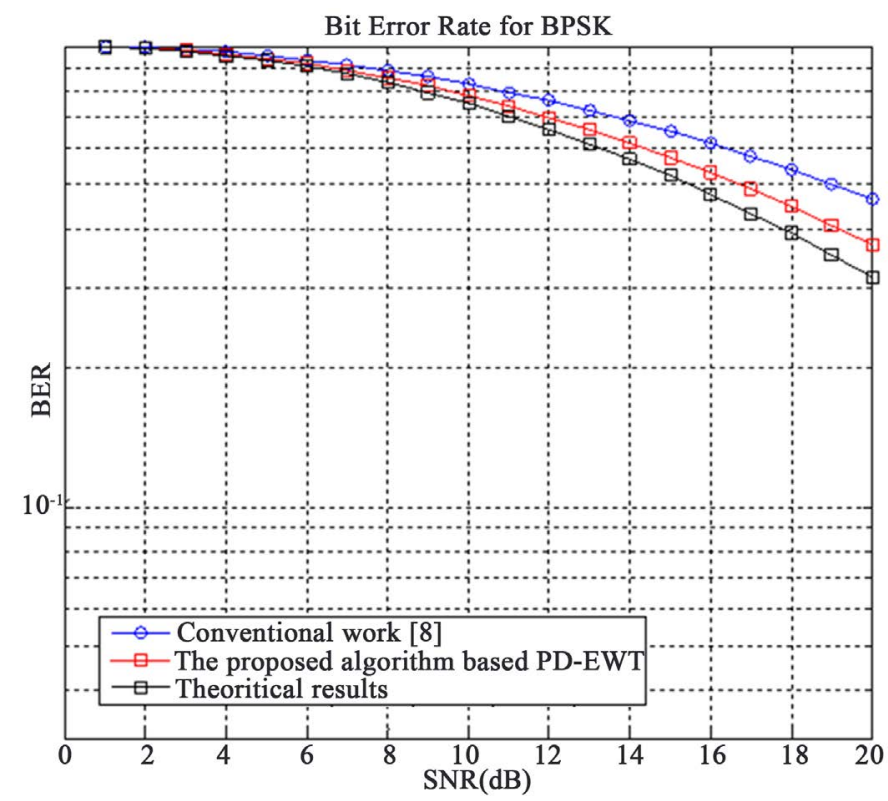

Figure 9. Compared BER curves for different reduction technique using case study II.

Table 2. Compared simulation results among different PAPR reduction techniques for different case studies.

\begin{tabular}{|c|c|c|c|c|c|c|c|c|}
\hline \multirow{2}{*}{\multicolumn{2}{|c|}{ Modulation Technique }} & \multicolumn{4}{|c|}{ PAPR (dB) } & \multicolumn{3}{|c|}{ Additional Reduction (\%) } \\
\hline & & No Coding & Based SLM & Based on [8] & Based on [9] & SLM & Convolutional Coding & Work [9] \\
\hline \multirow{3}{*}{16 QAM } & Case study I & & 3.6 & 3.5 & 2.7 & 72.1 & 44.5 & 11 \\
\hline & Case study II & 7.9 & 3.81 & 2.73 & 1.92 & 81.62 & 60 & 15 \\
\hline & Case study III & & 4.2 & 3.9 & 3.1 & 65.6 & 35.9 & 8 \\
\hline \multirow{3}{*}{ BPSK } & Case study I & & 5.6 & 4.1 & 3.7 & 51.6 & 32.3 & 9.8 \\
\hline & Case study II & 12.3 & 6.4 & 3.5 & 3.2 & 62 & 51.43 & 14 \\
\hline & Case study III & & 6.9 & 4.7 & 4.2 & 42.9 & 26.5 & 9 \\
\hline
\end{tabular}


This work contains three case studies based on the entropy level of the DWT. Thus, a comparison among PD-EWT, our previously published work, and the SLM has been made. The results show that we can use just the first two detailed parameters where the decomposition status after that is not accepted. From this comparison, the PD-EWT shows extraordinary promising results in allocating and combating the high peaks. The achieved performance improvement for allocating and combating the effect of the PAPR is between $8 \%-81 \%$ over the limitations that have been taken into consideration. Moreover, the BER for the best scenario has been reduced to $36 \times 10^{-2}$ from $48 \times 10^{-2}$ that has been achieved in our previously work.

\section{References}

[1] Lu, B., Wang, X. and Narayanan, K.R. (2002) LDPC-Based Space-Time Coded OFDM Systems over Correlated Fading Channels. IEEE Transaction .on Communications, 50, 74-88. http://dx.doi.org/10.1109/26.975756

[2] Ten Brink, S., Kramer, G. and Ashikhmin, A. (2004) Design of Low-Density Parity Check Codes for Multi-Antennas Modulation and Detection. IEEE Transaction .on Communications, 52, 670-678. http://dx.doi.org/10.1109/TCOMM.2004.826370

[3] Gonzalez-Rodriguez, J., Drygajlo, A., Ramos-Castro, D., Garcia-Gomar, M. and Ortega-Garcia, J. (2006) Robust Estimation, Interpretation and Assessment of Likelihood Ratios in Forensic Speaker Recognition. Computer Speech and Language, 20, 331-355. http://dx.doi.org/10.1016/j.csl.2005.08.005

[4] Juntti, M., Vehkapera, M., Leinonen, J., Zexian, V., Tujkovic, D., Tsumura, S. and Hara, S. (2005) MIMO MCCDMA Communications for Future Cellular Systems. IEEE Communication Magazine, 43, 118-124.

[5] Abdullah, Kh. and Hussain, Z.M. (2009) Studies on DWT-OFDM and FFFT-OFDM Systems. International Conference on Communication, Computer and Power (Icccp’09), Muscat, 15-18 February 2009.

[6] Han, S. and Lee, J. (2004) PAPR Reduction of OFDM Signals Using a Reduced Complexity PTS Technique. IEEE Signal Processing Letters, 11, 887-890. http://dx.doi.org/10.1109/LSP.2004.833490

[7] Ben Aicha, A., Tilli, F. and Ben Jebara, S. (2004) PAPR Analysis and Reduction in WPDM Systems. IEEE First International Symposium on Control, Communications and Signal Processing, 315-318.

[8] Daoud, O., Hamarsheh, Q. and Al-Sawalmeh, W. (2012) Enhancing the BER of MIMO-OFDM Systems for Speaker Verification. IEEE SSD13 Conference, Tunisia, 18-21 March 2013, 1-6.

[9] Al-Akaidi, M., Daoud, O. and Linfoot, S. (2007) A new Turbo Coding Approach to Reduce the Peak-to-Average Power Ratio of a Multi-Antenna-OFDM. International Journal of Mobile Communications, 5, 357-369. http://dx.doi.org/10.1504/IJMC.2007.012399

[10] Tellado, J. (2002) Multicarrier Modulation with Low PAR Applications to DSL and Wireless. Kluwer Academic Publishers, New York.

[11] Zheng, X., Sun, M. and Tian, X. (2008) Wavelet Entropy Analysis of Neural Spike Train. IEEE Congress on Image and Signal Processing, 1, 225-227. http://dx.doi.org/10.1109/cisp.2008.530

[12] Gray, R. (1990) Entropy and Information Theory. Springer, New York. http://dx.doi.org/10.1007/978-1-4757-3982-4

\section{Submit or recommend next manuscript to SCIRP and we will provide best service for you:}

Accepting pre-submission inquiries through Email, Facebook, LinkedIn, Twitter, etc.

A wide selection of journals (inclusive of 9 subjects, more than 200 journals)

Providing 24-hour high-quality service

User-friendly online submission system

Fair and swift peer-review system

Efficient typesetting and proofreading procedure

Display of the result of downloads and visits, as well as the number of cited articles

Maximum dissemination of your research work

Submit your manuscript at: http://papersubmission.scirp.org/ 